\section{The necessity for}

T-cryptantigen activation screening in babies with necrotising enterocolitis

\author{
G. F. Kirsten, J. Smith, C. Pieper, A. Bird, \\ G. Wessels, S. Riphagen, S. Moore
}

Objective. To determine the prevalence of T-cryptantigen activation (TCA) and its predictive value for severity of necrotising enterocolitis (NEC) in babies.

Study design. Prospective descriptive.

Study population. Thirty-four babies with NEC were prospectively screened for TCA at Tygerberg Hospital over a 6-month period. TCA screening was done by testing for red blood cell agglutination by the common peanut lectin, Arachis hypogea. Once TCA was confirmed, only washed red cells were administered to the babies and plasmacontaining blood products were avoided. NEC was divided into suspected NEC (stage 1), classic NEC (stage 2) and fulminant NEC (stage 3).

Main outcome measures. Prevalence of TCA in babies with various stages of NEC; the association between TCA and bowel necrosis, need for surgery and mortality.

Results. TCA was positive in $8(24 \%)$ of the babies in this study. Six babies (18\%) had stage 1 NEC, $10(29 \%)$ had stage 2 NEC and $18(53 \%)$ had fulminating or stage 3 NEC. All 18 babies with stage 3 NEC required surgery and TCA was present in $8(47 \%)$ of them. Twelve babies $(35 \%)$ died, 3 with TCA and 9 with no TCA. Babies with TCA had portal venous gas on abdominal radiographs $(P=0.037)$ and stage 3 NEC $(P=0.003)$ more often than babies with no TCA.

Conclusions. A strong association was noted between TCA and the fulminant form of NEC with bowel necrosis. TCA in a baby with NEC should alert the surgeon to the possibility of severe disease and the need to avoid plasma-containing blood products.

Departments of Paediatrics and Paediatric Surgery, Tygerberg Hospital and University of Stellenbosch, Tygerberg, W. Cape

G. F. Kirsten, M.MED. (PAED.), FC.P. (S.A.), PAED. D.C.H. (S.A.)

J. Smith MMED PAED.)

C. Pieper, MMED. PAED.

G. Wessels M MED, PAED,

S. Riphagen, M.B. CH.E

S. Moore, F.R.C.S. (EDIN.), M.D.

Western Province Blood Transfusion Service, Cape Town

A. Bird, M.MED PATH. (HAEM,), FF, PATH, (HAEM)
Blood banks are urged to introduce routine screening for TCA in all babies with NEC.

S Afr Med J 1996; 86: 546-548.

It is often very difficult to determine early which babies with necrotising enterocolitis (NEC) need surgery. T-cryptantigen activation (TCA), which has been associated with the fulminant form of NEC,' may therefore be important for its predictive value in this regard. TCA's tendency to precipitate acute intravascular haemolysis ${ }^{2}$ after plasma-containing blood product transfusion has far-reaching implications for the management of these babies. It therefore seems essential that all babies with suspected NEC be screened for TCA. Such routine screening was recently introduced at Tygerberg Hospital in order to determine the prevalence of TCA and its predictive value for outcome in babies with NEC.

We report on the babies with NEC who were screened for TCA over a 6-month period.

\section{Patients and methods}

All babies with NEC treated in the neonatal wards at Tygerberg Hospital were prospectively screened for TCA from August 1993 to January 1994.

NEC was diagnosed on the clinical picture of feeding intolerance, abdominal distension, bloody stools and radiological demonstration of pneumatosis intestinalis, or by means of histological evidence of NEC in a surgical pathology specimen. The severity of NEC was graded according to the modified criteria of Bell (as modified by Walsh and Kliegman) $)^{3}$ where stage 1 NEC constituted suspected NEC, stage 2 classic NEC (the presence of pneumatosis intestinalis on plain abdominal radiograph in a moderately ill baby) and stage 3 fulminating NEC with peritonitis, perforation or portal venous gas on radiograph.

Once NEC was suspected, red cells from the babies were tested for TCA. Blood samples from affected patients were screened by testing for red blood cell agglutination with the common peanut lectin, Arachis hypogea. ${ }^{4}$ One drop of a saline extract of this anti-T lectin (prepared from raw unsalted shelled peanuts) is added to one drop of red cells, mixed well and incubated at room temperature for 15 minutes. After centrifugation, the tubes are read macro- and microscopically for agglutination. Positive controls (prepared by treating fresh group $O$ red blood cells with neuraminidase) as well as negative controls are set up with each test. Blood cultures were done in all patients while peritoneal fluid cultures were done if appropriate. Initial antibiotic management consisted of penicillin, amikacin and metronidazole. Indications for surgical intervention included the presence of bowel perforation, peritonitis or clinical deterioration.

Patients with TCA were only given washed red cells; plasma-containing blood products such as fresh-frozen plasma were avoided.

Clotting abnormalities were managed with vitamin $K$, cryoprecipitate and platelets when the platelet count was $<40 \times 10^{\circ} /$. 
Student's $t$-test and the chi-square test were used to determine statistical significance $(P \leqslant 0.05)$.

\section{Results}

Altogether 34 babies with NEC were diagnosed over a 6 -month period. The mean birth weight of the babies was $1416.3 \mathrm{~g}$ (range 655 - $3240 \mathrm{~g}$ ), mean gestational age 31.4 weeks (range 26 - 40 weeks) and mean onset of NEC 13 days (range 2 - 59 days). Six (18\%) had stage 1, 10 (29\%) stage 2 and $18(53 \%)$ stage 3 NEC. Twenty-two babies $(65 \%)$ survived. Eight babies $(44 \%)$ with stage 3 NEC had TCA while none with stages 1 and 2 was affected (sensitivity $44 \%$, specificity $100 \%$, positive predictive value $100 \%$, negative predictive value $62 \%$ ). The clinical characteristics and outcome of the babies with the various stages of NEC are shown in Table I. All 18 babies with stage 3 NEC required surgery within 24 hours because of the onset of symptoms of acute peritonitis. In 3 , abdominal drains were inserted. Four of the 7 babies with stage 3 NEC who had portal venous gas on abdominal radiographs had TCA, while only 1 baby with NEC (TCA-negative) had radiological evidence of free air in the abdomen. Bowel necrosis with perforation occurred in all 8 babies with TCA and all required surgery.

Table I. The characteristics and outcome of babies with various stages of NEC

\begin{tabular}{|c|c|c|c|c|}
\hline & $\begin{array}{l}\text { Stage } \\
1\end{array}$ & $\begin{array}{l}\text { Stage } \\
2\end{array}$ & $\begin{array}{l}\text { Stage } \\
3\end{array}$ & $\begin{array}{l}\text { All } \\
\text { patients }\end{array}$ \\
\hline No. $(\%)$ & $6(18 \%)$ & $10(29 \%)$ & $18(53 \%)$ & 34 \\
\hline Mean birth weight $(\mathrm{g})$ & 1188.3 & 1319 & 1546.1 & 1416.3 \\
\hline Mean gestational age (wks) & 30.7 & 30.6 & 32.0 & 31.4 \\
\hline \multicolumn{5}{|l|}{ Abdominal radiograph } \\
\hline Portal air & 0 & 0 & $7(39 \%)$ & $7(21 \%)$ \\
\hline Free air in abdomen & 0 & 0 & $1(6 \%)$ & $1(3 \%)$ \\
\hline \multicolumn{5}{|l|}{ Surgery } \\
\hline Laparotomy & 0 & 0 & $15(63 \%)$ & $15(44 \%)$ \\
\hline Abdominal drain & 0 & 0 & $3(17 \%)$ & $3(9 \%)$ \\
\hline Positive blood culture & $2(33 \%)$ & $3(30 \%)$ & $7(39 \%)$ & $12(35 \%)$ \\
\hline \multicolumn{5}{|l|}{ Babies with positive } \\
\hline blood culture who died & 0 & $2(66 \%)$ & $3(43 \%)$ & $5(42 \%)$ \\
\hline TCA-positive & 0 & 0 & $8(44 \%)$ & $8(24 \%)$ \\
\hline \multicolumn{5}{|l|}{ Mean age at onset of } \\
\hline NEC (d) & 8.7 & 13.4 & 16.4 & 14.17 \\
\hline Died & 0 & $3^{\star}(30 \%)$ & $9(50 \%)$ & $12(35 \%)$ \\
\hline
\end{tabular}

* One baby died from nosocomially acquired candida septicaemia at 3 weeks of age.

Blood cultures were positive in $12(35 \%)$ of the babies. Of the babies with TCA, only $1(13 \%)$ had a positive blood culture (Enterobacter cloaca), while $11(42 \%)$ babies without TCA had positive blood cultures. Of these, $4(33 \%)$ grew Escherichia coli, 5 (42\%) Klebsiella pneumoniae, 1 a $\beta$ haemolytic streptococcus and 1 Staphylococcus epidermidis.

Three $(37 \%)$ of the babies with TCA died, while $9(35 \%)$ with no TCA died. Of the 12 babies who died, 5 (41\%) (all TCA-negative) had a positive blood culture. Another baby died after 3 weeks from a nosocomially acquired candida septicaemia.

The characteristics of the babies with and without TCA are shown in Table II.
Table II. Characteristics of babies with NEC and TCA v. no TCA

\begin{tabular}{llll}
\hline Characteristic & TCA & No TCA & $P$ \\
\hline Total & 8 & 26 & \\
Mean birth weight (g) & 1750 & 1313 & 0.1475 \\
& $($ SD 748) & $($ SD 294) & \\
Mean gestational age (wks) & 33.6 & 30.7 & 0.0544 \\
& (SD 3.6) & $($ SD 2.1) & \\
Mean age at onset of NEC (d) & 18.4 & 12.9 & 0.2997 \\
& $($ SD 15.0) & $($ SD 12.2) & \\
No. with stage 3 NEC & $8(100 \%)$ & $10(38 \%)$ & 0.003 \\
No. with venous portal gas & 4 & 3 & 0.037 \\
No. with positive blood culture & 1 & 11 & 0.210 \\
Survivors & 5 & 17 & 1.000 \\
Survivors with stage 3 NEC & 5 & 4 & 0.637 \\
Babies with positive blood & & & \\
Cultures who died & 0 & 5 & 1.000 \\
\hline
\end{tabular}

\section{Discussion}

NEC is a common condition of premature babies affecting approximately $12 \%$ of very-low-birth-weight infants ${ }^{5}$ $(<1500 \mathrm{~g})$. The reported mortality rate ranges between $30 \%$ and $40 \%$, but may be as high as $80 \%$ in babies weighing less than $1000 \mathrm{~g} .{ }^{6}$ The mortality rate is also directly related to the severity of NEC and is highest in babies with stage 3 disease. Novak et al. ${ }^{\prime}$ demonstrated a decrease in mortality in babies with NEC after the introduction of routine screening for TCA activation and the avoidance of transfusion of plasma-containing blood products in TCA babies. Given the bias towards more severely affected infants in the TCA group, the $35 \%$ mortality rate in these babies in the present series might have been even greater had the use of plasma-containing products not been avoided.

Thomsen-Friedenreich cryptantigens (T-cryptantigens) are naturally occurring antigens normally concealed by neuraminic acid in the sub-terminal layer of the red blood cell membrane. ${ }^{4.7}$ Certain bacteria such as clostridia, bacteroides, Streptococcus pneumoniae and certain viruses produce neuraminidase which may destroy the neuraminic acid on the surface of the red blood cells, thus exposing the hidden cryptantigens - so-called TCA. ${ }^{4.7}$ This renders the red cells polyagglutinable when exposed to cryptantigen antibodies. These antibodies are naturally acquired haemagglutinins (IgM subclass) found in most humans after the age of 6 months and are, therefore, present in most plasma-containing blood products. ${ }^{7.8}$

TCA occurs in $11-34 \%$ of babies with severe NEC, ${ }^{7}$ a finding comparable to the $24 \%$ noted in the present series.

The strong association between TCA and a fulminant course of NEC with bowel necrosis and a high risk of intestinal perforation ${ }^{7}$ was confirmed in our study: babies with stage 1 and 2 NEC were TCA-negative, while 8 babies with stage 3 NEC and TCA had bowel necrosis with perforation. TCA in babies with NEC should alert the surgeon to the severity of the disease and the probability of early operative intervention. ${ }^{9}$

Intrahepatic portal venous gas in babies with NEC, as demonstrated on abdominal radiograph, has been associated with extensive bowel necrosis and a high 
mortality rate. ${ }^{2}$ Portal venous gas was present in 4 (50\%) of the babies with TCA and in $3(12 \%)$ with no TCA. All these patients were shown to have extensive bowel necrosis.

Three of the babies with TCA died (38\%). Of these, 1 had severe bronchopulmonary dysplasia (BPD) and died from complications associated with BPD. The other 2 babies were more mature ( 36 and 40 weeks). One developed fulminating NEC after severe birth asphyxia and an exchange transfusion for rhesus incompatibility, while the other, referred to us on day 4 , had bowel obstruction caused by an undiagnosed imperforate anus.

Bacteria are associated with NEC, but their role is as yet unclear. ${ }^{5}$ Anaerobic bacteria such as clostridia are often associated with a more fulminant disease course. ${ }^{5}$ One of the babies with TCA had a positive blood culture, while a variety of aerobic organisms was cultured from the blood of the babies with no TCA.

No anaerobic bacteria were cultured from either the blood or peritoneal fluid of any of the babies, despite correct sample collection techniques. This may be related to the great difficulty experienced by microbiology laboratories in culturing anaerobes. ${ }^{\circ}$ Four of the babies $(80 \%)$ with blood cultures positive for Klebsiella died, even in the presence of stage 2 NEC. The clinical course of these infants was severely influenced by septicaemia-related complicatons, especially pneumonia.

We advise that screening for TCA activation be performed routinely in babies with NEC. ${ }^{2}$ The screening test is simple, quick and inexpensive to perform. ${ }^{4}$ Arachis hypogea strongly agglutinates T-activated red cells. ${ }^{7.10}$

Positive tests should be quantified, as the degree of activation correlates with the likelihood of haemolysis following transfusion of plasma products as well as with the severity of NEC.

If the test is positive, the clinician should be informed so that only washed red cells or platelets are used and immunoglobulin-containing products such as plasma are avoided.

A major predicament is the need for plasma-derived products in the resuscitation of infants with stage 3 NEC, especially if their condition is complicated by an acquired coagulopathy. These products contain cryptantibodies and may precipitate massive intravascular haemolysis in infants with TCA.

General supportive measures, such as fluid replacement using colloids or albumin for treatment of shock, are advised. Specific treatment of the underlying condition is of major importance and may be the only measure needed for mild disseminated intravascular coagulation (DIC). If surgery is planned or the patient is actively bleeding, replacement transfusion therapy with washed red cells, platelets and (if available) cryoprecipitate may be undertaken. In infants with severe DIC the safest measure is the transfusion of plasma previously identified to have a low titre of cryptantibodies. ${ }^{7.1}$ If this is not available we propose that a double-volume exchange transfusion with washed cells suspended in albumin or, if technically feasible, plasmapheresis using albumin as replacement fluid, will be a more effective method of treatment than transfusion with plasma-derived (high cryptantibody titre) replacement products. If a baby with TCA requires an exchange transfusion, the washed erythrocytes should be suspended in $5 \%$ albumin. ${ }^{12}$
Screening for TCA in babies should be undertaken routinely when NEC is first suspected. It is important to realise that TCA cannot be detected by standard crossmatching techniques.

In infants with NEC, TCA is transient and parallels the course of the disease but may persist for as long as 120 days after resolution of NEC. ${ }^{7}$ For this reason screening must be continued on a weekly basis until TCA is negative and must be repeated prior to any plasma-containing blood product transfusion.

In summary, this study confirms the strong association between TCA, the fulminant form of NEC and bowel necrosis. The finding of TCA in a baby with NEC should alert the surgeon to the possibility of a severe degree of NEC requiring surgery. The administration of plasma-containing blood products will further increase the morbidity and possibly mortality of these babies. The routine screening for TCA in babies with NEC should constitute an integral part of their management.

The authors would like to thank Miss G. van Alphen and the technicians of the Western Province Blood Transfusion Service at Tygerberg Hospital for performing the cryptantigen screening, and Miss E. Marais for typing the manuscript.

\section{REFERENCES}

1. Novak AW, Abbott AE, Klein RL. T-cryptantigen determination affects mortality and morbidity in necrotizing enterocolitis. Surg Gynecol Obstet 1993; 176: 368-370. Stringer MD. Spitz L. Surgical management of necrotising enterocolitis. Arch Dis Child 1993; 69: 269-270,

Walsh M. Kliegman AM. Necrotizing enterocolitis: treatment based on staging criteria. Pediatr Clin North Am 1986; 33: 179-201

Novak AW. Bacterial-induced ABC alterations complicating necrotizing enterocolitis. Am J Dis Child 1984; 138: 183-185.

Caplan MS, Mackendrick W. Necrotizing enterocolitis. A review of pathogenetic mechanisms and implications for prevention. Pediatr Pathol 1993; 13: $357-369$ . Neonatal Care. 3rd ed. Boston: Little Brown and Company, 1991: 511-517.

7. Rodwell A, Tudehop DI. Screening for cryptantigen exposure and polyagglutination in neonates with suspected necrotizing enterocolitis. J Paediatr Child Health 1993 29: $16-18$.

a. Kim YD. Prakash U. Webster GF. Hargie M. Nature of human serum blood group antibodies. Immunol Commun 1979; 8: 397-406.

Klein RL, Novak RW, Novak PE. T-cryptantigen exposure in neonatal necrotizing enterocolitis. J Pediatr Surg 1986: 21: 1155-1158.

11 Sivergieid A Hafleign washed platelet concentrates in patients with non-haemolytic transfusion reactions. Transfusion 1977: 17: 33-37.

12. Mupanemunda $\mathrm{AH}$, Kenyon CF, Inwood MJ. Leigh K. Bacterial-induced activation of erythrocyte t-antigen complicating necrotizing enterocolitis: a case report. Eur Of erythrocyte t-antigen compli

Accepted 4 Jul 1994 